nebulizer). However, the adsorption rate in Figure $2 \mathrm{C}$ is still more rapid than that of vortexed CLL dispersions in Figure 1 for the uniform final dispersion concentration (5 mg CLL/71 ml subphase) used (see "Materials and Methods"). This dilute concentration was chosen because it allows a discrimination between the adsorption isotherms of CLL $\left(S_{i}\right)$ and CLL (V). At higher concentrations $(\geq 0.25 \mathrm{mg} / \mathrm{ml}$ ) vortexed CLL dispersions adsorb as rapidly as the sonicated dispersions in Figure 2.

29. If the assumption is made that all of the aerosolized solution (a total of $1.5 \mathrm{ml}$ in 30 minutes containing $0.15 \mathrm{M} \mathrm{NaCl}$ ) is retained by the infant, this would result in a sodium load of about $0.25 \mathrm{mEq}$. Note that the surfactant dose calculations here are for material which is actually deposited at the alveolar level (20\% of inhaled amount). In animal studies of surfactant replacement $(22,23)$, typical tracheal instillation doses are 5-10 times larger than in this example calculation. However, a significant percentage of instilled surfactant probably does not reach the alveolar interface.

30. This paper is based on work performed under Contract No. DE-ACO2. 76EV03490 with the U.S. Department of Energy at the University of Rochester Department of Radiation Biology and Biophysics and has been assigned Report No. UR-3490-2213. This work was also supported in part by NIH grants HL-25170 and K04-HL-00945. L. Marks was the recipient of a summer research fellowship at the University of Rochester School of Medicine (HHS grant HL-07496). The assistance of Dr. Richard Taubold with surface property measurements is gratefully acknowledged.

31. Requests for reprints should be addressed to: R. H. Notter, Department of Pediatrics, Division of Neonatology, University of Rochester School of Medicine, Rochester, New York 14642.

32. Received for publication October $22,1982$.

33. Accepted for publication March 9, 1983.

\title{
Pseudomonas-Infected Cystic Fibrosis Patient Sputum Inhibits the Bactericidal Activity of Normal Human Serum
}

\author{
NEAL L. SCHILLER ${ }^{(30)}$ AND RANDAL L. MILLARD \\ Division of Biomedical Sciences and Department of Biology, University of California, Riverside, California, USA
}

\section{Summary}

Sputum samples were obtained from nimeteen cystic fibrosis (CF) patients with respiratory tract infections due to Pseudomonas aeruginosa and were examined for the presence of a specific local protective or blocking factor, which might partially explain the inability of CF patients' pulmonary defense mechanisms to clear their lungs of Pseudomonas infection. Using an assay that measures the bactericidal activity of fresh normal human serum (FHS), eighteen of nineteen CF sputa examined were capable of protecting either autologous or heterologous strains of $P$. aeruginosa from serum bactericidal activity. Most of this protective activity was absorbable at $4^{\circ} \mathrm{C}$ by Pseudomonas, and could be reduced by dilution in phosphate buffered saline or counteracted by an increase in serum concentration. This protective activity is believed to be due to a Pseudomonas-specific bactericidal blocking antibody. In contrast, only two of 17 non-CF sputa were found to significantly inhibit the bactericidal activity of FHS for $P$. aeruginosa. Furthermore, three out of five CF sputa also protected Escherichia coli from the bactericidal activity of FHS. This protective activity was much less than that observed with $P$. aeruginosa, and was not absorbable at $4^{\circ} \mathrm{C}$, suggesting the presence of a second blocking or protective factor in CF sputum. From these observations, we conclude that sputum from CF patients infected with $\boldsymbol{P}$. aeruginosa contains one or more factors that interfere with the bactericidal activity present in FHS.

\section{Abbreviations}

BHI, brain heart infusion

CF, cystic fibrosis

CFU, colony forming unit

FHS, fresh normal human serum

PBS, phosphate buffered saline

PIA, pseudomonas isolation agar

sIgA, secretory IgA
Chronic obstructive pulmonary infection is the major cause of morbidity and mortality in people with cystic fibrosis (CF). Although respiratory infection with Staphylococcus aureus often occurs, Pseudomonas aeruginosa (particularly the mucoid colonial form) eventually emerges as the dominant pathogen in $70-90 \%$ of CF patients, and $>90 \%$ die of progressive pulmonary insufficiency complicated by infection with $P$. aeruginosa (4).

Except for respiratory tract infections, CF children are not more susceptible to infection than normal children of comparable age. $\boldsymbol{P}$. aeruginosa is known to be an opportunistic pathogen, which commonly infects the debilitated patient $(25,26)$, and is seldom isolated from pediatric patients with chronic respiratory disease other than CF $(4,11)$. These basic clinical observations, coupled with the fact that pulmonary infections rarely spread outside the respiratory tract in CF patients, strongly suggest a defect in local pulmonary defense mechanisms.

Despite the presence of elevated levels of Pseudomonas antibodies in CF patients' sera and pulmonary secretions $(13,15)$, these bacteria are not cleared by pulmonary defense mechanisms. Alveolar macrophages isolated from CF patients appear normal (23), but CF serum appears to interfere with the ability of rabbit and human alveolar macrophages to ingest and destroy Pseudomonas $(2,3,5,21,23)$. This effect has been attributed to either a serum deficiency (2), or a serum inhibitory factor $(3,21,23)$. Fick et al. (5) have recently demonstrated that $\mathrm{IgG}$ antibodies from CF patients are impaired in their attachment to the Fc receptors of human alveolar macrophages.

In addition to interfering with phagocytosis, CF serum has also been found to block serum bactericidal activity for $P$. aeruginosa. Whereas $P$. aeruginosa strains isolated from CF patients were more sensitive to the bactericidal activity of normal human serum than strains isolated from non-CF patients (12, and Schiller, N.L., manuscript in preparation), sera from CF patients chronically infected with $\boldsymbol{P}$. aeruginosa showed a selective inability in bactericidal activity against the patient's own $P$. aeruginosa isolate, 
suggesting the presence of "bactericidal blocking" antibodies (12). A similar heat-stable blocking factor has been detected in the serum of patients with chronic $P$. aeruginosa infection and characterized as $\operatorname{IgG}(8,24)$. Recently, Thomassen and Demko (22) identified the presence of a protective or blocking factor in sera from CF patients in poor clinical condition, and have suggested that the presence of this factor may contribute to the failure of $\mathrm{CF}$ patients to control infection.

The purpose of the present study was to examine the sputum of chronically infected CF patients for the presence of a serum bactericidal blocking factor. We reasoned that because infection with $P$. aeruginosa remains localized in the respiratory tract and is not cleared by pulmonary defense mechanisms, a factor(s) that protects this microorganism from host defenses should be present in respiratory secretions. The results suggest that CF sputum contains one or more factors that interfere with the bactericidal activity of normal human serum.

\section{MATERIALS AND METHODS}

Sputum samples. Sputum samples from nineteen CF patients colonized with $P$. aeruginosa were obtained through the generous cooperation of the following individuals: Dr. Richard Dooley, Director of the Brian Wesley Ray CF Center, San Bernardino County Medical Center, San Bernardino, CA; Dr. Chun-I Wang, Director of the CF Center, Children's Hospital of Los AngelesUSC School of Medicine; and Dr. Alan Osher, Director of the CF Center, UCLA Medical Center, Los Angeles. CF patients ranged in age from 7-37 years (average age $=18.6$ years) and included 10 females and nine males. Their clinical conditions and duration of Pseudomonas colonization/infection are presented in Table 1 . The clinical evaluation of patients was based on the ShwachmanKulcyzki (20) scoring system, in which a maximal score of 25 points is awarded for each of four categories-chest roentgenogram, general activity, physical examination, and nutrition-for a best possible score of 100 points. Patients were classified by their physician into four categories as follows: (1) good clinical condition, total score of $80-100$; moderate clinical condition, total score of 60-80; advanced clinical condition, total score of 40-60; and poor clinical condition, total score of $<40$. Sputum samples from seventeen non-CF patients (hospitalized with a variety of clinical conditions) were randomly selected and generously sup. plied by Drs. John Lewis and Ira Roy, Clinical Microbiology Laboratory, Loma Linda University Medical Center, Loma Linda, CA. Samples of sputum were kept frozen until ready for use.

Preparation of sputum samples. For these studies, each sample of sputum was first plated onto BHI Agar (BBL Microbiology Systems, Cockeysville, MD) and PIA (Difco Laboratories, Detroit MI) to isolate any bacterial species present. The sputum was then separated into the soluble and gel phases by emulsifying the sputum with a tissue homogenizer and centrifuging the emulsion at $120,000 \times g$ for $4 \mathrm{~h}$ at $4^{\circ} \mathrm{C}$. (Occasionally, a sputum sample had to be diluted first with PBS to provide enough volume for sample preparation). The supernatant was then filtered through a $0.45 \mu \mathrm{m}$ filter and stored in small aliquots at $-70^{\circ} \mathrm{C}$.

Bacterial cultures. All strains of $P$. aeruginosa employed in these studies were isolated by this laboratory from the sputum of CF patients, and identified by gram-stain, oxidase reaction, pigment production, and growth on PIA and at $42^{\circ} \mathrm{C}$. Where required, further identification was accomplished using Flow Laboratories, Inc. N/F System (Flow Laboratories, Inc., Roslyn, NY). In addition, a mucoid strain of Escherichia coli was isolated from a CF patient sputum and generously provided to us by the Clinical Microbiology Laboratory at San Bernardino County Medical Center.

Serum bactericidal/blocking assay. Blood was obtained from five normal adult male volunteers (who have not been previously infected with $P$. aeruginosa) by venipuncture and allowed to clot at room temperature for $30 \mathrm{~min}$. After centrifugation at $1000 \times \mathrm{g}$ for $15 \mathrm{~min}$ at $4^{\circ} \mathrm{C}$, the serum was removed, pooled and either used immediately or stored at $-70^{\circ} \mathrm{C}$ in small aliquots until ready for use. For this assay, Pseudomonas strains were grown overnight in BHI broth at $37^{\circ} \mathrm{C}$, adjusted spectrophotometrically at $550 \mathrm{~nm}$ to a concentration of approximately $1 \times 10^{8} \mathrm{CFU} / \mathrm{ml}$ and then diluted in $0.01 \mathrm{M}$ PBS, pH 7.4 to a final concentration of $5 \times 10^{4}$ $\mathrm{CFU} / \mathrm{ml}$.

The following studies were done using 96-well tissue culture plates (Becton Dickinson and Co., Oxnard, CA). To each well was added $100 \mu$ l of either PBS or sputum (undiluted or serially diluted in PBS). After adding $50 \mu \mathrm{l}$ of bacteria $\left(5 \times 10^{4} \mathrm{CFU} / \mathrm{ml}\right)$, the mixtures were incubated at $37^{\circ} \mathrm{C}$ for $30 \mathrm{~min}$ with gentle rotation using a Lab-Line Orbit Environ-Shaker (Lab-Line Instruments, Inc., Melrose Park, IL). Although preliminary experiments had suggested that this preincubation step increased blocking activity, further studies revealed that preincubation was not necessary for most experiments. Nevertheless for continuity with earlier experiments, all of the experiments described herein utilized this preincubation step.

After this preincubation step, $100 \mu \mathrm{l}$ of either $3.13 \%$ FHS or PBS was added, and the mixtures were reincubated at $37^{\circ} \mathrm{C}$ for 60 min with gentle rotation. Afterwards, the tissue culture plate was placed on ice to stop any further reaction, and $100 \mu \mathrm{l}$ and $10 \mu \mathrm{l}$ aliquots were removed from each well and plated onto BHI agar, incubated overnight at $37^{\circ} \mathrm{C}$ and the number of $\mathrm{CFU} / \mathrm{ml}$ determined. As controls for each experiment, bacteria were incubated in PBS alone, in PBS with FHS, and in PBS with sputum. The number of bacteria in sputum + FHS was then compared to the number of bacteria in the sputum + PBS control, and all values were then computed as \% bacterial survival according to the following formula:

$$
\frac{\mathrm{CFU} \text { in (Sputum }+ \text { FHS) }}{\mathrm{CFU} \text { in (Sputum }+ \text { PBS) }} \times 100 \%=\% \text { survival }
$$

Absorption experiments. Overnight BHI broth grown cultures (at $37^{\circ} \mathrm{C}$ with agitation) were centrifuged at $7000 \times \mathrm{g}$ for $5 \mathrm{~min}$; the pellet was resuspended in PBS and adjusted spectrophotometrically to a concentration of approximately $1 \times 10^{9}$ organisms $/ \mathrm{ml}$. For each $\mathrm{ml}$ of sputum to be absorbed, $3 \times 10^{9}$ organisms were centrifuged at $7000 \times g$ for $5 \mathrm{~min}$, the supernate discarded, and the pellet resuspended in sputum to yield a final concentration of approximately $3 \times 10^{9} \mathrm{bacteria} / \mathrm{ml}$ sputum. This mixture was then tumbled end-over-end for $60 \mathrm{~min}$ at $4^{\circ} \mathrm{C}$. Afterwards, the mixture was centrifuged at $7000 \times g$ for $5 \mathrm{~min}$, and the absorbed supernatant was then used to resuspend a second bacterial pellet and the absorption repeated. After the third absorption, the bacteriasputum mixture was then centrifuged at $25,000 \times g$ for $60 \mathrm{~min}$ and the supernatant was filter sterilized through a $0.45 \mu$ filter. This absorbed sputum was either used immediately or stored in small aliquots at $-70^{\circ} \mathrm{C}$ until ready for use. As a control, a sputum sample without bacteria was handled similarly and labeled as absorbed sputum control.

\section{RESULTS}

In a study to be described in detail elsewhere (Schiller, N. L., and $\mathbf{R}$. A. Hatch, manuscript in preparation), the sensitivity of mucoid and nonmucoid strains of Pseudomonas aeruginosa to the bactericidal activity of FHS was compared. Whereas $88.5 \%$ of mucoid strains $(23 / 26)$ were sensitive to $\leq 1.56 \%$ FHS (i.e., $>90 \%$ of the organisms were killed at this serum concentration after incubation for $60 \mathrm{~min}$ at $37^{\circ} \mathrm{C}$ ), only $14.8 \%$ of nonmucoid strains $(9 / 61)$ were sensitive to $\leq 1.56 \%$ FHS. To ensure the detection of serum bactericidal blocking activity in CF sputum samples, only highly serum-sensitive mucoid strains (obtained from the sputum of CF patients) were employed in the studies described below.

The ability of CF sputum to protect $P$. aeruginosa from the bactericidal activity of FHS was examined. In each of these experiments, the survival of the test strain in the test sputum is first determined (CFU in $40 \%$ sputum/CFU in PBS); the number of CFU in sputum then serves as the control for determining the $\%$ survival of the test strain in the presence of FHS and sputum 


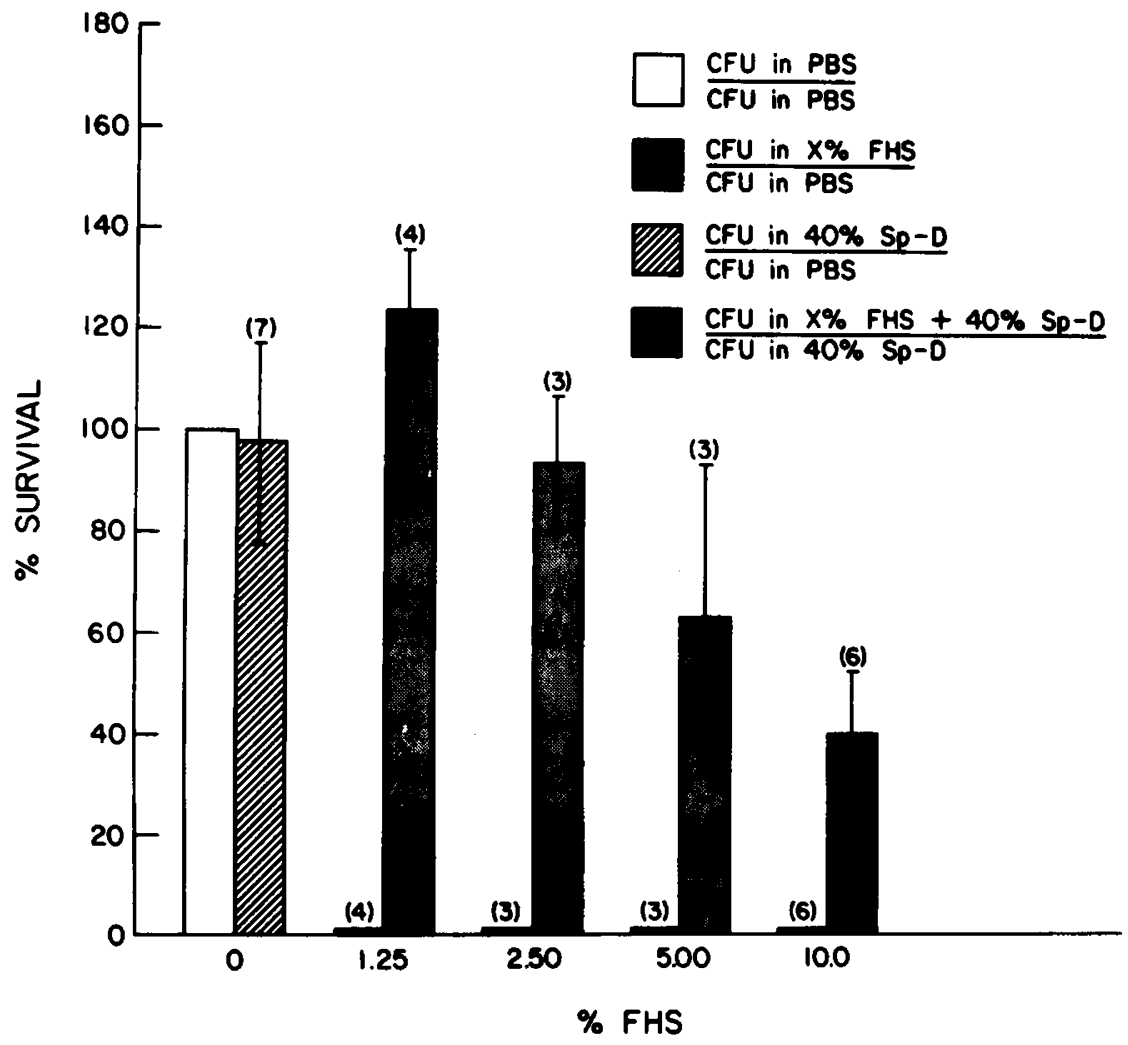

Fig. 1. Effect of $40 \%$ Sp-D sputum on the bactericidal activity of normal fresh human serum (FHS). The survival of bacterial strain Ps-D was examined in the presence of increasing concentrations of FHS in the presence or absence of $40 \%$ Sp-D sputum. Values represent the mean \pm standard error of mean based on the number of experiments shown in parentheses.

(CFU in sputum + FHS/CFU in sputum + PBS). The results obtained using one CF sputum preparation are shown in Figure 1. FHS, at concentrations of $1.25 \%$ or higher, was bactericidal for Ps-D, a strain isolated from Sp-D sputum, with less than $1 \%$ survival routinely observed. The addition of $40 \% \mathrm{Sp}-\mathrm{D}$ sputum completely blocked the killing activity of $1.25 \%$ FHS for Ps-D. This blocking activity was reduced when the serum concentration was raised from 1.25 to $10 \%$ FHS (as shown here), or when the sputum concentration was diluted in PBS (as illustrated in Figure 3 with Sp-K sputum).

Further studies were performed to determine whether this protective activity in CF sputum was limited to the strain(s) isolated from that particular sputum. For these experiments, the blocking activity of four CF sputa, one non-CF sputum (infected with a nonmucoid strain of $P$. aeruginosa, Ps-L), and one non-CF, non$P$ seudomonas infected sputum (from a patient with allergic bronchitis) were examined using a battery of five test strains. Each of these strains was highly serum-sensitive in the absence of sputum. The results are presented in Figure 2. Although in a few cases a particular sputum protected its autologous strain better than other strains (e.g., Sp-D and Ps-D), all five strains were protected from the bactericidal activity of FHS by each of these four sputa. The only exception was that $\mathrm{Sp}-\mathrm{K}$ was directly bactericidal for Ps-I in the absence of FHS. In contrast to these CF sputa, Sp-M, the non$\mathrm{CF}$, non-Pseudomonas infected sputum did not protect any strain tested. Sp-L, the non-CF, Pseudomonas-infected sputum showed slight protective activity specifically for Ps-K. (Note: Ps-L was not serum-sensitive and could not be employed in this assay).

Nineteen CF sputum samples were obtained, and one or more mucoid strains were isolated from 15 of these sputa. All nineteen CF sputa were then examined for blocking activity using either an autologous or heterologous Pseudomonas strain, and 18 of these CF sputa $(94.7 \%)$ exhibited significant protective activity (i.e., > $20 \%$ bacterial survival). The results are shown in Table 1 . The blocking activity of 7 CF sputa for their autologous mucoid isolates is shown in the top portion of this table, and averaged
$100.2 \%$ bacterial survival. The bactericidal blocking activity of the remaining 12 CF sputa was examined using heterologous strain Ps-P which was used for these studies because four of these sputa did not harbor a mucoid Pseudomonas strain in the sputum sample examined (perhaps due to antibiotic chemotherapy) and the remaining five sputa were colonized/infected with a mucoid strain which was phenotypically unstable. The blocking activity of these 12 CF sputa are shown on the bottom portion of Table 1 (average $64.2 \%$ bacterial survival). Only one $\mathrm{CF}$ sputum was considered to be not significantly protective, Sp-T (12.6 $\pm 9.79 \%)$. It is not known if this sputum would have been significantly protective had higher sputum concentrations been available for examination. From this Table, it would appear that blocking activity is not directly related to clinical condition, because high levels of blocking activity were observed in patients in $\operatorname{good}(\mathrm{Sp}-\mathrm{Q})$ as well as poor clinical condition (Sp-D).

In contrast to the results with CF sputa, only two of 17 non-CF sputa examined for blocking activity using strain Ps-P were found to be significantly protective (i.e., $>20 \%$ bacterial survival). Both of these sputa were heavily infected with Staphylococcus aureus and averaged $96.8 \%$ bacterial survival. Two non-CF, Pseudomonas-infected sputa were only slightly protective for Ps-P (average - 9\% bacterial survival). Of the remaining 13 non-CF sputa, four had no blocking activity, and 9 were slightly protective (average $=12.2 \%$ bacterial survival).

Five CF sputum samples (Sp-D, Sp-K, Sp-N, Sp-O, Sp-P) were also examined for protective activity for a mucoid strain of Escherichia coli isolated from a CF sputum. Three of the sputa (Sp-D, $\mathrm{Sp}-\mathrm{N}$ and $\mathrm{Sp}-\mathrm{O}$ ) demonstrated blocking activity for this strain. This protective activity for $E$. coli, however, was usually much less than that for $\boldsymbol{P}$. aeruginosa. For example, the \% survival of $\boldsymbol{P}$. aeruginosa strain Ps-N incubated in serum $+\mathrm{Sp}-\mathrm{O}$ sputum was $79.2 \pm 13.6 \%$, whereas the \% survival of $E$. coli incubated in serum $+\mathrm{Sp}-\mathrm{O}$ sputum was only $16.9 \pm 5.3 \%$. Without Sp-O sputum, however, there was no survival of either strain in $1.25 \%$ FHS.

Most of the protective activity present in CF sputum was 


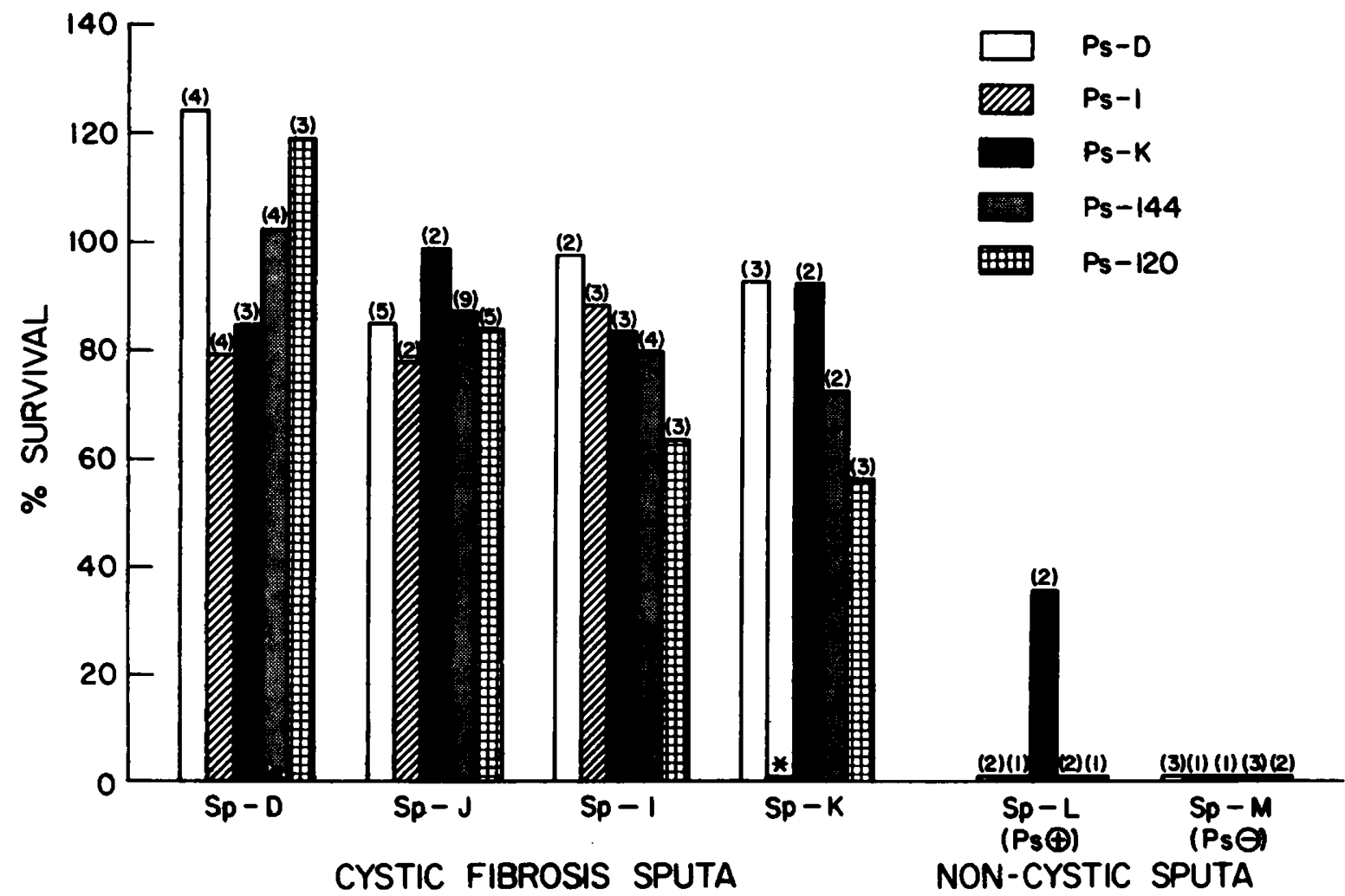

Fig. 2. Comparison of Pseudomonas protective activity in CF and non-CF sputa for five $P$. aeruginosa strains. The values represent the mean $\%$ survival of each strain in the presence of $1.25 \%$ normal fresh human serum and $40 \%$ of the indicated sputum. Numbers in parentheses indicate the number of experiments done in each case. * = sputum was bactericidal for Ps-I without addition of serum.

Table 1. Ability of CF sputum to protect autologous or heterologous mucoid strains of $P$. aeruginosa from the bactericidal activity of $1.25 \%$ fresh normal human serum $(F H S)^{1}$

\begin{tabular}{|c|c|c|c|c|}
\hline Sputum $^{2}$ & $\begin{array}{c}\text { Clinical } \\
\text { condition }^{3}\end{array}$ & $\begin{array}{l}P . \text { aeruginosa } \\
\text { culture positive }\end{array}$ & $\begin{array}{c}\text { Test } \\
\text { strain }\end{array}$ & $\%$ Survival $^{5}$ \\
\hline Sp-D & Poor & $>10 \mathrm{yr}$ & Ps-D & $123.7 \pm 11.6$ \\
\hline$S p-N$ & Poor & $>10 \mathrm{yr}$ & Ps-N & $110.1 \pm 24.5$ \\
\hline Sp-E & Advanced & $>7 \mathrm{yr}$ & Ps-E & $98.6 \pm 25.4$ \\
\hline Sp-I & Moderate & Intermittent $-7 \mathrm{yr}$ & Ps-I & $88.4 \pm 15.6$ \\
\hline Sp-K & Moderate & $>10 \mathrm{yr}$ & Ps-K & $112.0 \pm 19.2$ \\
\hline Sp-P & Moderate & $>7 \mathrm{yr}$ & Ps-P & $53.4 \pm 7.9$ \\
\hline Sp-Q & Good & $>7 \mathrm{yr}$ & Ps-Q & $115.2 \pm 21.6$ \\
\hline Sp-A & Poor & $>4 \mathrm{yr}$ & Ps-P & $71.8 \pm 6.6$ \\
\hline Sp-S & Poor & $>10 \mathrm{yr}$ & Ps-P & $45.6 \pm 9.7$ \\
\hline Sp-C & Poor & $>6 \mathrm{yr}$ & Ps-P & $93.7 \pm 9.0$ \\
\hline Sp-F & Poor & $>10 \mathrm{yr}$ & Ps-P & $35.6 \pm 11.5$ \\
\hline Sp-G & Poor & $>6 \mathrm{yr}$ & Ps-P & $37.9 \pm 11.3$ \\
\hline $\mathbf{S p}-\mathbf{H}^{6}$ & Poor & $>10 \mathrm{yr}$ & Ps-P & $98.7 \pm 12.1$ \\
\hline Sp-J & Advanced & $>10 \mathrm{yr}$ & Ps-P & $93.6 \pm 6.6$ \\
\hline Sp-B & Moderate & $>6 \mathrm{yr}$ & Ps-P & $119.2 \pm 17.4$ \\
\hline Sp-O & Moderate & $>10 \mathrm{yr}$ & Ps-P & $48.7 \pm 2.2$ \\
\hline Sp-R & Moderate & $>10 \mathrm{yr}$ & Ps-P & $65.7 \pm 11.1$ \\
\hline Sp-T & Moderate & $>10 \mathrm{yr}$ & Ps-P & $12.6 \pm 9.7$ \\
\hline Sp-U & Good & $>7 \mathrm{yr}$ & Ps-P & $47.6 \pm 20.2$ \\
\hline
\end{tabular}

${ }^{1}$ All strains tested were sensitive to $1.25 \%$ FHS, i.e., less than $1 \%$ survival was routinely observed.

${ }^{2}$ All sputa were tested at a final concentration of $40 \%$, except for $S p-G$, Sp-R, and Sp-U (20\% sputum) and Sp-S and Sp-T (10\% sputum).

'Clinical condition is defined in "Materials and Methods" section of the text.

${ }^{4}$ Refers to the numbers of years that this patient had $P$. aeruginosa positive cultures.

${ }^{6}$ Values represent the mean \pm standard error of mean based on at least three experiments each.

${ }^{6} \mathrm{Sp}$-H was directly bactericidal for Ps-P until dialyzed extensively against PBS. Values here reflect blocking activity of dialyzed Sp-H. determined to be removal by absorption of sputum with $P$. aeruginosa. A representative experiment is shown in Figure 3. The protective activity of Sp-K sputum for Ps-K was removed by preabsorption with Ps-K as shown using sputum concentrations of $20 \%$ or less. At $40 \%$ sputum, however, the absorbed and unabsorbed sputum samples were equally protective. Similar results were obtained when Sp-K was absorbed with Ps-D and when Sp-N was absorbed with Ps-N. In contrast, absorption of Sp-N or Sp-O with $E$. coli did not reduce the protective activity of these sputa either for $P$. aeruginosa strains or for $E$. coli. Furthermore, absorption of Sp-N with Ps-N did not reduce the protective activity of this sputum for $E$. coli although it significantly reduced the protective activity for Ps-N.

\section{DISCUSSION}

The inability of CF patients to clear their lungs of $P$. aeruginosa infection, despite the presence of normal alveolar macrophages (23) and elevated levels of Pseudomonas antibodies in their sera and pulmonary secretions $(13,15)$, has prompted the search for a specific blocking factor(s) which might explain these observations. Whereas most studies have focused on CF sera, we chose to examine CF sputum to determine if a specific local protective or blocking factor could be detected there. In addition we decided to use an assay which measures the bactericidal activity of FHS for mucoid strains of $P$. aeruginosa. This bactericidal activity is believed to be mediated primarily by IgG and/or IgM and the classical complement pathway (N. L. Schiller and M. J. Alazard, manuscript in preparation). This serum bactericidal assay was chosen for several reasons: (1) the essay can be performed with relatively small amounts of patient material; (2) one can monitor blocking activity affecting either or both antibody- or complement-mediated functions; and (3) the knowledge that a serum bactericidal blocking factor(s) has previously been suggested or demonstrated to be present in CF serum $(8,12,22,24)$.

Eighteen out of nineteen CF sputa examined were capable of significantly inhibiting the serum bactericidal activity of FHS for either autologous or heterologous strains of $P$. aeruginosa. In a 


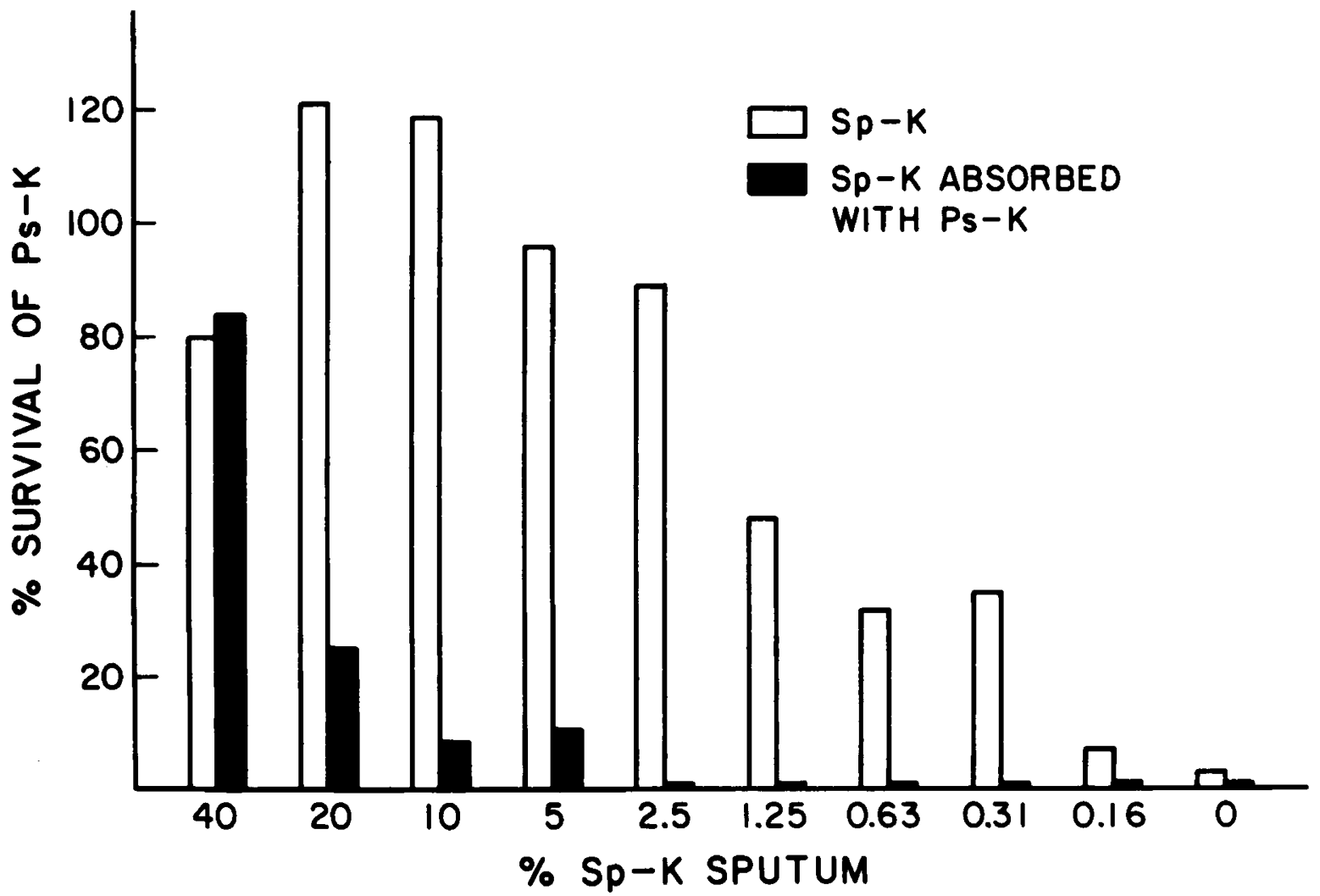

Fig. 3. Blocking activity in CF sputum is removable by absorption with $P$. aeruginosa. The \% survival of Ps-K in $1.25 \%$ FHS with or without Sp-K sputum (in concentrations ranging from 40 to $0.16 \% \mathrm{Sp}-\mathrm{K}$ ) was determined before and after absorption of sputum with Ps-K.

few cases the best protection was demonstrated with an autologous sputum-mucoid isolate combination, but in general this protective activity was usually nonstrain specific for Pseudomonas. In contrast, only two of 17 non-CF sputa demonstrated comparable blocking activity for $P$. aeruginosa. In a few cases, $40 \%$ CF sputum was directly cidal for a specific Pseudomonas strain. This cidal activity was usually only observed at the highest sputum concentrations, and protective activity could often be observed at $20 \%$ sputum concentrations or below, or after the sputum had been exhaustively dialyzed with PBS.

When considering the probable serum bactericidal blocking factors in CF sputum, at least three possibilities come to mind. First, CF sputum might harbor blocking antibodies-sIgA, IgA, IgM, or IgG-that might bind to the bacterial cell surface and compete with serum bactericidal immunoglobulins. Several investigators have previously delineated the existence of "bactericidal blocking" antibodies $(8,12,22,24)$ characterized as $\operatorname{IgG}(8,24)$ in CF serum. Second, CF sputum might contain bacterial cellular components-slime, lipopolysaccharide, outer membrane proteins, etc.-that might tie up serum bactericidal antibodies and fix complement (i.e., immune complexes). There have been several reports of immune complexes, both in sera $(1,14)$ and sputum $(18)$ of CF patients. Finally, CF sputum might have bacterial or host-derived enzymes-protease, elastase, lipase, etc.-that might inactivate complement or degrade the bactericidal immunoglobulin target(s) on the bacterial cell surface. It should be noted that Schultz and Miller (19) have reported that the Pseudomonas extracellular enzyme, elastase, can directly inactivate complement.

Our results seem to implicate the presence of at least two serum bactericidal blocking factors. Most, but not all, the blocking activity in CF sputum was absorbable at $4^{\circ} \mathrm{C}$ with Pseudomonas. This blocking activity could be reduced by dilution of sputum with PBS or counteracted by raising the serum concentration from 1.25 to $10 \%$ or higher. These observations suggest that this blocking factor can attach to the bacterial surface and can compete with the bactericidal factor(s) present in FHS. We believe that this factor is a Pseudomonas-specific bactericidal blocking antibody. It should be noted that all of the CF sputum samples examined in these studies were obtained from patients chronically colonized/ infected with $P$. aeruginosa and hence expected to contain antiPseudomonas immunoglobulins $(13,15)$. The presence of $\operatorname{IgA}$ and IgG antibodies against surface antigens of $P$. aeruginos $a$ in sputum has been previously described (17). Furthermore, Hann and Holsclaw (10) demonstrated that all strains of $P$. aeruginosa recovered from sputum specimens of CF patients were coated with antibodies of IgA, IgG, and IgM classes. Of specific interest was their observation that the most intense coating of the bacterial surface was with IgA. Because IgA does not ordinarily fix complement or promote phagocytosis, its binding to bacterial surfaces may interfere with the binding of bactericidal IgG or IgM antibodies. IgA has been shown to block the binding of $\operatorname{IgG}$ to antigen $(7,9)$ and the complement-mediated lysis of Neisseria meningitidis (6) and IgG-sensitized erythrocytes (16), possibly by sterically hindering the deposition of activated complement components on the target cells. Considering the fact that sIgA would be expected to be elevated in these infected CF sputum samples, and its potential for inhibiting serum bactericidal reactions, we suggest that part of the Pseudomonas protective activity in CF sputum is due to "bactericidal blocking" antibodies of the sIgA class. The specificity of these "bactericidal blocking" antibodies might also explain the higher levels of blocking activity observed when autologous sputa and mucoid isolate combinations were used. Preliminary studies in this laboratory have detected blocking activity in a columnfractionated sputum pool containing sIgA but not IgG (N. L. Schiller, unpublished observations). Further studies to characterize this blocking factor in CF sputum are in progress. In addition, it will be of interest to determine if this blocking factor can also interfere with the opsonization and/or phagocytosis of $P$. aeruginosa by alveolar macrophages. Several investigators have previously described such blocking activity in CF sera $(2,3,5,21,23)$.

In three of five CF sputa, $E$. coli was also slightly protected from serum bactericidal activity, although at a much lower level than $P$. aeruginosa. Unlike the $P$. aeruginosa protective factor, however, this blocking activity was not absorbable at $4^{\circ} \mathrm{C}$. This might suggest the presence of a second factor, which nonspecifically inhibits antibody and complement-mediated bacteriolysis. 
The presence of a second factor might also explain the residual protective activity for $\boldsymbol{P}$. aeruginosa observed in CF sputum after absorption with Pseudomonas, as well as the low-level blocking activity observed in eleven non-CF sputa (it may not explain, however, the two non-CF sputa with significant blocking activity). Preliminary studies in this laboratory have demonstrated that the incubation of CF sputum with FHS for $60 \mathrm{~min}$ at $37^{\circ} \mathrm{C}$ markedly reduces the complement hemolytic ${ }_{50}$ titers of FHS (N. L. Schiller and R. L. Millard, unpublished observations). Similar results were obtained when filter-sterilized culture filtrates of $P$. aeruginosa were incubated with FHS (N. L. Schiller and R. L. Millard, unpublished observations). It is not known whether this complement depletion represents complement activation and consumption (either through antibody-antigen-complement activation or direct antigen-complement activation) or complement degradation (such as direct complement enzymatic inactivation). Further studies are needed to establish the identity and source of this second blocking factor in CF sputum, and to determine if it is related to the complement depleting activity detected in Pseudomonas culture filtrates.

In summary, eighteen of nineteen sputum samples obtained from CF patients chronically colonized/infected with $P$. aeruginosa were found to contain one or more factors which interfered with the bactericidal activity of FHS. One factor was absorbable by $P$. aeruginosa and is believed to be a Pseudomonas-specific bactericidal blocking antibody. The presence of Pseudomonas protective activity in CF sputum might be expected to interfere with pulmonary defense mechanisms and partially explain why CF patients are usually unable to clear this microorganism from their lungs.

\section{REFERENCES AND NOTES}

I. Berdischewsky, M., Pollack, M., Young, L. S., Chia, D., Osher, A. B., and Barnett, E. V.: Circulating immune complexes in cystic fibrosis. Pediatr. Res. 14: 830 (1980).

2. Biggar, W. D., Holmes, B., and Good, R. A.: Opsonic defect in patients with cystic fibrosis of the pancreas. Proc. Natl. Acad. Sci., 68: 1716 (1971)

3. Boxerbaum, B., Kagumba, M., and Mathews, L. W.: Selective inhibition of phagocytic activity of rabbit alveolar macrophages by cystic fibrosis serum. Am. Rev. Respir. Dis., 108: 777 (1973).

4. Di Sant'Agnese, P. A. and Davis, P. B.: Research in cystic fibrosis (Part 3). N. Engl. J. Med., 295: 597 (1976).

5. Fick, R. B., Jr., Naegel, G. P., Matthay, R. A., and Reynolds, H. Y.: Cystic fibrosis Pseudomonas opsonins. Inhibitory nature in an in vitro phagocytic assay. J. Clin. Invest., 68: 899 (1981).

6. Griffiss, J. McL: Bactericidal activity of meningococcal antisera: blocking by IgA of lytic antibody in human convalescent sera. J. Immunol., 114: 1779 (1975).

7. Griffiss, J. McL. and Bertram, M. A.: Immunoepidemiology of meningococcal disease in military recruits. II. Blocking of serum bactericidal activity by circulating IgA early in the course of invasive disease. J. Infect. Dis., 136: 733 (1977).

8. Guttman, R. M. and Waisbren, B. A.: Bacterial blocking activity of specific IgG in chronic Pseudomonas aeruginosa infection. Clin. Exp. Immunol., 19: 121 (1975).

9. Hall, W. H., Manion, R. E., and Zinneman, H. H.: Blocking serum lysis of Brucella abortus by hyperimmune rabbit immunoglobulin A. J. Immunol. 107: 41 (1971).
10. Hann, S. and Holsclaw, D. S.: Interactions of Pseudomonas aeruginosa with immunoglobulins and complement in sputum. Infect. Immun., 14:114 (1976).

11. Hoiby, N.: Pseudomonas aeruginosa infection in cystic fibrosis. Acta Pathol. Microbiol. Scand. Sect. C, 262: 1 (1977).

12. Hoiby, N. and Olling, S.: Pseudomonas aeruginosa infection in cystic fibrosis bactericidal effect of serum from normal individuals and patients with cystic fibrosis on $P$. aeruginosa strains from patients with cystic fibrosis or other diseases. Acta Pathol. Microbiol. Scand. Sect. C, 85: 107 (1977).

13. May, J. F., Herrick, N. C., and Thompson, D.: Bacterial infection in cystic fibrosis. Arch. Dis. Child., 47: 908 (1972).

14. Moss, R. B. and Lewiston, N. J.: Immune complexes and humoral response to Pseudomonas aeruginosa in cystic fibrosis. Am. Rev. Respir. Dis., 121: 23 (1980).

15. Neter, E.: Pseudomonas aeruginosa infection and humoral antibody response of patients with cystic fibrosis. J. Infect. Dis., 130 (Suppl.): S132 (1974).

16. Russell-Jones, G. J., Ey, P. C., and Reynolds, B. C.: The ability of IgA to inhibit the complement-mediated lysis of target red blood cells sensitized with IgG antibody. Mol. Immunol., 117: 1173 (1980).

17. Schiotz, P. O., Hoiby, N., Permin, H., and Wiik, A.: IgA and IgG antibodies against surface antigens of Pseudomonas aeruginosa in sputum and serum from patients with cystic fibrosis. Acta Pathol. Microbiol. Scand. Sect. C, 87: 229 (1979).

18. Schiotz, P. O., Nielsen, H., Hoiby, N., Glikmann, G., and Svehag, S.-E.: Immune complexes in the sputum of patients with cystic fibrosis suffering from chronic Pseudomonas aeruginosa lung infection. Acta Pathol. Microbiol. Scand. Sect. C, 86: 37 (1978).

19. Schultz, D. R. and Miller, K. D.: Elastase of Pseudomonas aeruginosa: inactivation of complement components and complement-derived chemotactic and phagocytic factors. Infect. Immun., 10: 128 (1974).

20. Shwachman, H., Kulczycki, L. L., and Khaw, K. T.: Studies in cystic fibrosis: a report on sixty-five patients over 17 years of age. Pediatrics, 36: 6891965.

21. Thomassen, M. J., Boxerbaum, B., Demko, C. A., Kuckenbrod, P. J., Dearborn, D. G., and Wood, R. E.: Inhibitory effect of cystic fibrosis serum on Pseudomonas phagocytosis by rabbit and human alveolar macrophages. Pediatr. Res. I3: 1085 (1979).

22. Thomassen, M. J. and Demko, C. A.: Serum bactericidal effects on Pseudomonas aeruginosa isolates from cystic fibrosis patients. Infect. Immun., 33: 512 (1981).

23. Thomassen, M. J. Demko, C. A. Wood, R. E., Tandler, B., Dearborn, D. G. Boxerbaum, B., and Kuchenbrod, P. J.: Ulstructure and function of alveolar macrophages from cystic fibrosis patients. Pediatr. Res., 14: 715 (1980)

24. Waisbren, B. A. and Brown, I.: A factor in the serum of patients with persisting infection that inhibits the bactericidal activity of normal serum against the organism that is causing the infection. J. Immunol., 97: 431 (1966).

25. Wood, R. E.: Pseudomonas: the compromised host. Hosp. Pract., 11: 91 (1976).

26. Young, L. S. and Armstrong, D.: Pseudomonas aeruginosa infections. CRC Crit. Rev. Clin. Lab. Sci., 3: 291 (1972).

27. This paper is warmly dedicated to the memory of Randal Lee Millard whose bright future was tragically cut short by a motorcycle accident on January 27, 1982.

28. The authors gratefully acknowledge the excellent technical assistance of Joseph M. Marietta and Patricia Balazs. The authors also acknowledge the generous cooperation and support of Richard Dooley, M.D., and Chun-I Wang, M.D., who not only contributed patient material for this study but also contributed their time and effort towards the successful completion of this study.

29. This research was supported in part by Public Health Service grant AI-17072 from the National Institutes of Allergy and Infectious Diseases, Public Health Service Biomedical Research Development Grant RR 09070 and Biomedical Research Support grant RR 05816 from the National Institutes of Health, a grant from the American Lung Association of California, and the Randal Lee Millard Memorial Fund for Cystic Fibrosis Research.

30. Requests for reprints should be addressed to: Dr. Neal L. Schiller, Division of Biomedical Sciences and Department of Biology, University of California, Riverside, CA 92521.

31. Received for publication September 2, 1982

32. Accepted for publication March 10, 1983. 